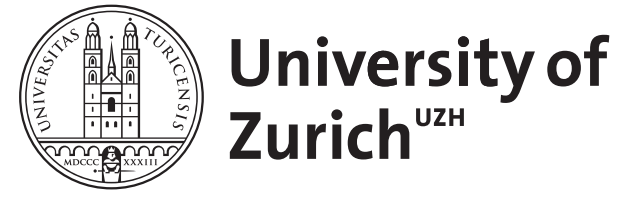
Archive

University of Zurich

University Library

Strickhofstrasse 39

CH-8057 Zurich

www.zora.uzh.ch

Year: 2016

Mittelalterliches Code-Switching? Zu den Sprachwechselstrategien Notkers III. von St. Gallen

Glaser, Elvira

DOI: https://doi.org/10.1515/jbgsg-2016-0004

Posted at the Zurich Open Repository and Archive, University of Zurich ZORA URL: https://doi.org/10.5167/uzh-150088

Journal Article

Published Version

Originally published at:

Glaser, Elvira (2016). Mittelalterliches Code-Switching? Zu den Sprachwechselstrategien Notkers III. von St. Gallen. Jahrbuch für Germanistische Sprachgeschichte, 7:35-60.

DOI: https://doi.org/10.1515/jbgsg-2016-0004 
Elvira Glaser

\section{Mittelalterliches Code-Switching? Zu den Sprachwechselstrategien Notkers III. von St. Gallen}

\section{Die, Mischprosa‘ Notkers III.}

Notker III., der St. Galler Mönch und Schulmeister, ist in der deutschen Sprachgeschichte bekannt als Urheber einer umfangreichen volkssprachlichen Überlieferung, die zum einen in der großen Mehrzahl ihren Ausgangspunkt in einer lateinischen Vorlage hat, also eine Übersetzung aus dem Lateinischen darstellt, und zum anderen in unterschiedlichem Umfang innerhalb althochdeutscher Passagen lateinische Elemente aufweist. Die so entstandenen komplexen Textensembles, die in der Regel aus lateinischer Vorlage, althochdeutscher Übersetzung und fallweise lateinischen und althochdeutschen Kommentaren bestehen, sind in schulischem Kontext entstanden, wie Ekkehart IV. von St.Gallen in seinem Liber benedictionum ausführt ${ }^{1}$, und sie wurden wohl für ein vertieftes Studium der lateinischen Quellentexte benutzt. Das komplexe Textarrangement hat immer wieder auch Editoren vor enorme Herausforderungen gestellt, wenn sie die verschiedenen Bestandteile graphisch und im Layout erkennbar machen wollten, wie das an dem folgenden Beispiel eines Notker'schen Psalmenkommentars illustriert werden soll, $\mathrm{zu}$ dessen differenzierter Darstellung auf verschiedene Schriftauszeichnungen (kursiv, fett, unterstrichen, Farbe) zurückgegriffen wird:

1 Ekkehart (V. 62) schreibt über Notker III.: Teutonice propter caritatem discipulorum plures libros exponens, ,aus Liebe zu seinen Schülern hat er mehrere Bücher auf deutsch übersetzt und erläutert".

Anmerkung: Der vorliegende Beitrag ist aus Vorträgen zu verschiedenen Anlässen an den Universitäten Utrecht, München und Bonn erwachsen. Den jeweiligen Diskussionsteilnehmern bin ich ebenso zu Dank verpflichtet wie den Studierenden des Zürcher Seminars „Diglossie und Sprachmischung bei Notker von St. Gallen“ (2013). Für verschiedene Hinweise danke ich Andreas Nievergelt und Paul Widmer, Zürich.

Elvira Glaser, Zürich/Schweiz 
Et erit tanquam lignum quod plantatum est secus decursus aquarum. Unde der gediêhet also uuóla. so der bôum. der bîdemo rínnenten uuazzere gesezzet ist. Quod fructum suum dabit in tempore suo. Der zîtigo sînen uuûocher gíbet. Daz rinnenta uuazzer ist gratia sancti spiritus gnâda des hêiligen gêistis. Den sî nezzet. ter ist pírig pôum gûotero uuercho

Et folium eius non defluet. Noh sîn lôub ne rîset. Taz chît. noh sîn uuórt ne uuirt uuéndig. Et omnia quecumque faciet prosperabuntur. Unde frám diêhent álliû. diu der boum bíret unde bringet. ioh fructus. ioh folia in mêino facta \& dicta.

Abb. 1: Ps. 1,3 (St. Gallen, Stiftsbibliothek, Cod. Sang. 21)

Der lateinische Text (Psalm 1,3) Et erit tanquam lignum quod plantatum est secus decursus aquarum. Quod fructum suum dabit in tempore suo. Et folium eius non defluet. Et omnia quaecumque faciet prosperabuntur. ${ }^{2}$ ist in der Wiedergabe des Notker'schen Textes in kursiv erkennbar. ${ }^{3}$ Die schwarzen Passagen in Normalschrift zeigen Notkers Übersetzung. Notkers Kommentare sind als graue Passagen mit Unterstreichung erkennbar. Die lateinischen Elemente innerhalb des Kommentars sind (schwarz) fett markiert. Im Folgenden will ich nicht weiter auf die Gesamtanlage der Notker'schen Texte bzw. der überlieferten Handschriften eingehen, sondern den Blick insbesondere auf die in Althochdeutsch gehaltenen kommentierenden Passagen und die in diesen Passagen vorhandenen lateinischen Wörter und Phrasen richten. Die eigentlichen Übersetzungen sind, wie die bisherige Forschung bereits festgestellt hat, weitgehend von solchen lateinischen Elementen frei (Glauch 2000: 150).

Seit dem 19. Jahrhundert hat sich die Forschung intensiv mit Notkers Sprache beschäftigt. Mit Bezug auf die Gestaltung der althochdeutschen Textpassagen im Notker'schen Textarrangement wurde etwa von „Mischprosa“ gesprochen (vgl. die Titel Hoffmann 1910, Schaumann 1911), von „Pädagogenjargon“ (Hoffmann 1910: 4-5), und es wurden immer wieder Versuche unternommen, die Motivation der lateinischen „Einsprengsel“ $\mathrm{zu}$ ermitteln. So wurden sie etwa als „Residua“, stehengebliebenes Latein, charakterisiert (Kögel 1897: 614), in der Annahme, zunächst seien die Kommentare von Notker in Latein verfasst worden, unter Ver-

2 In Übersetzung: ,und er ist wie ein Baum, der an Wasserbächen gepflanzt ist, der zur rechten Zeit seine Frucht bringt. Und dessen Blattwerk nicht welkt. Alles, was er tut, wird ihm gut gelingen.'

3 Eine Auszeichnung des lateinischen Textes findet sich auch in der Einsiedler Handschrift (St. Gallen, Stiftsbibliothek, Cod. Sang. 21), in roter Tinte. Das ist allerdings nicht generell in den Notker-Handschriften üblich. Die weitere Differenzierung zwischen den althochdeutschen Textpartien und dem lateinischen Kommentar bietet auch die Psalmenhandschrift nicht. 
wendung älterer lateinischer Textkommentare, wie es für Notker ja hinreichend belegt wurde. Bekannt ist Georg Baeseckes Verdikt, Notkers Sprache sei ein „häßlicher Mischmasch“ (1950: 33). Stefan Sonderegger (2003: 137) sieht die „Reservate und Zitate“ als Strategie zur „Verankerung des althochdeutschen Textes im Lateinischen“. In jüngerer Zeit wurde weiter von einer negativen Beurteilung der Notker'schen Sprachenverwendung abgerückt. Sonja Glauch (2000: 154) spricht von einem speziellen „Duktus der Rede“, der hiermit gestaltet worden sei. Erst Anna Grotans hat einen Vergleich mit modernem Code-Switching explizit in die Diskussion eingebracht, insofern sie das Verfahren als ,situational“ code-switching (Grotans 2006: 153) klassifiziert, was die kontaktlinguistische Forschung der letzten Jahrzehnte als Charakteristikum bilingualer Gruppen herausgearbeitet hat. ${ }^{4}$

\section{Code-Switching-Forschung und historische Texte}

Es herrscht allerdings keine allgemeine Übereinstimmung darüber, was unter Code-Switching genau zu fassen ist und wie es sich etwa gegenüber Code-Mixing und Borrowing abgrenzen lässt. So bezeichnet Pieter Muysken (2000: 1) „cases where lexical items and grammatical features from two languages appear in one sentence“ als Code-Mixing, während das für Auer (1999: 328) keine Abgrenzung zwischen Code-Switching und Code-Mixing ergibt. Für Shana Poplack \& Nathalie Dion (2012: 309) gilt jedoch: ,incorporating it [the other-language item, E.G.] along with its associated grammatical properties, a process to which we have been referring as code-switching.“ Ein notorischer Streitpunkt sind dabei die Einzelwortentlehnungen, denen in morphologiearmen Sprachen wie dem Englischen nicht immer anzusehen ist, welche grammatischen Merkmale sie transportieren, wo also - entsprechend einer bestimmten Definition - Code-Switching endet und Entlehnung beginnt, wenn diese beiden Verfahren unter den „language interaction phenomena“, wie Muysken (2000: 23) den ganzen Komplex nennt, überhaupt abgegrenzt werden sollen. Handelt es sich bei dem other-language item um ein Einzelwort, kommt der Fall der Bewahrung der gebersprachlichen Grammatik nach Poplack \& Dion praktisch gar nicht vor: „this almost never happens with single words“ (2012: 309), vgl. auch Poplack (2013: 13). „[L]one other-language

4 Grotans (2006: 145-154) bietet auch eine ausführliche Besprechung der früheren Einschätzungen von Notkers ,Mischsprache‘. 
items tend to be borrowed“(2012: 279) erklären sie daher und „nonce borrowings are not code-switches“ (2012: 310). Die Qualifikation eines Einzelwortes als Entlehnung (borrowing) setzt nach Poplack \& Dion umgekehrt nicht voraus, dass es sich um häufige, habituelle Übernahmen aus einer anderen Sprache handelt, entscheidend ist die grammatische Integration. Muysken (2000: 69-95) hatte dagegen nach einer ausführlichen Diskussion der Abgrenzungskriterien dafür plädiert, CodeSwitching von Einzelwörtern als Möglichkeit nicht auszuschließen, insbesondere, wenn grammatische Markierungen wie Pluralmarker aus der Gebersprache vorhanden sind (2000: 79-80). Schon von jeher hatte sich mit diesem Problem der Abgrenzung die praktische Lexikographie auseinanderzusetzen, wenn sie entscheiden musste, ob Lexeme als Entlehnung gewertet werden und damit Eingang in ein Wörterbuch finden sollen. ${ }^{5}$ Allgemeingültige Kriterien scheint es hierfür nicht zu geben, wobei Frequenz und grammatische Integration entscheidende Faktoren sind, die aber in unterschiedlicher Weise gewichtet werden können.

Trotz aller terminologischen Unterschiede und Abgrenzungsprobleme gegenüber dem Vorgang der Entlehnung gibt es einen übereinstimmenden Kernbereich der Erforschung des intrasententiellen Wechsels zwischen lexikalischen oder grammatischen Elementen zweier verschiedener Sprachen. Der Fokus liegt hierbei auf der Ermittlung von Regeln und Beschränkungen für das Vorkommen von Elementen der betroffenen Sprachen in einem Satzzusammenhang. Muysken (2000) geht von verschiedenen Ausprägungen von Code-Switching bzw. Codemixing aus, die er als insertion, alternation und congruent lexicalization kategorisiert und mit den verschiedenen vorgeschlagenen Modellen zur Erfassung von Code-Switching, z.B. von Poplack und Carol Myers-Scotton, in Zusammenhang bringt. Darüber hinaus ist der intersententielle Wechsel natürlich für gesprächsund soziolinguistisch orientierte Forschungsfragen und für die Charakterisierung der Gesamtsituation des Code-Switchings ebenfalls von Interesse.

Mit der Qualifikation als Code-Switching ist Notkers Umgang mit Althochdeutsch und Latein in den Fokus kontaktlinguistischer Forschung gerückt. Bei der Betrachtung der Notker'schen Texte wird allerdings zu berücksichtigen sein, dass wir es mit geschriebener Sprache zu tun haben. Diese ist bislang erst selten und eher erst in jüngerer Zeit Gegenstand kontaktlinguistischer Forschung gewesen (vgl. dazu Sebba 2012). Sprachhistorische Untersuchungen zum Code-Switching, die aber bislang ebenfalls nicht sehr zahlreich sind, ${ }^{6}$ müssen sich zwangsläufig

5 Die Problemlage wird für das Althochdeutsche ausführlich in Bulitta 2014 erläutert.

6 Vgl. dazu den Überblick über bisherige Untersuchungen bei Kämmerer (2006: 37-45) und Schendl (2012) sowie die generellen Betrachtungen von Skaffari \& Mäkilähde (2014). Als ein Beispiel für eine umfassende Analyse eines frühmittelalterlichen, lateinisch-volkssprachlichen 
mit eventuellen Besonderheiten eines schriftlichen Code-Switching bzw. der Frage, wie mündliche und schriftliche Verfahren zusammenhängen, auseinandersetzen (Kämmerer 2006: 48-58). Einige einschlägige Beobachtungen zu Notker finden sich aus der Frühzeit der modernen kontaktlinguistischen Forschung in der Untersuchung Birgit Stolts zu Luthers Tischreden (1964: 272-281). ${ }^{7}$

Im Folgenden möchte ich nun nicht weiter der globalen Frage nachgehen, welche Art von Code-Switching in Notkers Sprachgebrauch möglicherweise dokumentiert ist und welche Funktion es hat, sondern mich in dem vorgegebenen Rahmen mit den konkreten language interaction phenomena, d.h. mit dem zweifellos vorliegenden abwechselnden Gebrauch von althochdeutschen und lateinischen Wörtern und Phrasen und den dabei dokumentierten sprachlichen Verfahren beim Aufeinandertreffen von althochdeutscher und lateinischer Grammatik beschäftigen. Was bisher bei der Betrachtung der Notker'schen Sprache nämlich weitgehend fehlt, ist eine kontaktlinguistisch inspirierte grammatische Analyse der von Notker verwendeten lateinischen Elemente. Eine solche Analyse ist nicht als Alternative zu einer funktionalen Fragestellung gedacht, sondern sie soll mit einem anderen Blick auf das Material die Einschätzung der Notker'schen Sprachwechselstrategien ergänzen. Angesichts des enormen Umfangs des Notker'schen Werkes kann hier allerdings nicht mehr als eine Pilotstudie vorgelegt werden, die anhand einer Auswahl einige grundsätzliche Beobachtungen macht und Fragen für die weitere Bearbeitung aufwirft, aber nicht den Anspruch erheben kann, Notkers Sprachgebrauch insgesamt zu charakterisieren.

\section{Analyse der Notkerschen ,Sprachmischung}

Meiner Fallstudie ist die Notker'sche Bearbeitung des Textes De nuptiis Philologiae et Mercurii von Martianus Capella zugrundegelegt, ${ }^{8}$ der Glauch (2000) eine umfassende Untersuchung gewidmet hat, die eine solche kontaktgrammatisch orientierte Auswertung enorm erleichtert. Ausgewertet wird zunächst die kommentierte althochdeutsche Übersetzung Notkers zu den 56 Kapiteln von Buch I. Dabei folge ich der Darstellung bei Glauch (2000), die Notkers Text inklusive Prolog in

Korpus unter dem Aspekt des Code-Switching ist v.a. Bisagni (2013/2014) zu nennen, eine detaillierte Analyse mittelenglischer Texte aus dem 15. Jahrhundert führt Schendl (2000) durch.

7 Stolt stützt sich für ihren kurzen Überblick auf Notkers Bearbeitung von Boethius' De consolatione Philosophiae (1964: 272).

8 In einer Abschrift aus dem 11. Jahrhundert in der Stiftsbibliothek St. Gallen überliefert und online konsultierbar: St. Gallen, Stiftsbibliothek, Cod. Sang. 872: De nuptiis Philologiae et Mercurii [http://www.e-codices.unifr.ch/de/list/one/csg/0872 (letzter Zugriff: 30.05.2016)]. 
440 Texteinheiten $^{9}$ gliedert, wovon knapp die Hälfte (218) keine lateinischen Elemente aufweist. Ich beziehe mich im Folgenden mit dem Terminus Abschnitt auf diese Texteinheiten. ${ }^{10}$ Die Identifikation lexikalischer Elemente als lateinisch wird durch Notkers Akzentsetzungssystem erleichtert, insofern man sich danach richten kann, dass von ihm als lateinisch angesehene Wörter keine Akzentzeichen erhalten.

Die Bandbreite zwischen den Kapiteln ist, sowohl was deren Umfang als auch was die Zahl lateinischer Einfügungen angeht, groß. Zum Beispiel finden sich in Kapitel 5431 Abschnitte, wovon nur drei einen - oder mehrere - lateinische Einschübe $^{11}$ aufweisen. Kapitel 18 hat nur neunAbschnitte, wovon aber sieben lateinische Einschübe enthalten. Eine Vorstellung vom durchschnittlichen Vorkommen lateinischer Einschübe vermittelt eine genauere Auszählung anhand der ersten acht Kapitel, die Glauch (2000) in 70 Abschnitte eingeteilt hat. Diese enthalten 114 lateinische Einschübe, also ein bis zwei Einschübe pro Abschnitt. Das spiegelt zum einen die Tatsache wider, dass Notker, wie oben erwähnt, bei der eigentlichen Übersetzung überwiegend keine lateinischen Wörter verwendet und damit Abschnitte, die nur aus der Übersetzung bestehen, z.B. I 1.5. und I 1.14., keine lateinischen Einschübe aufweisen. Aber auch dort, wo Notker althochdeutsche Kommentare hinzufügt, weisen diese Abschnitte keinesfalls regelmäßig lateinische Einschübe auf, so bleiben im ersten Kapitel etwa die Abschnitte 1.3., 1.4. und 1.11.-14. ohne Einschübe.

9 Definiert als „Sätze oder Satzabschnitte, wie sie von Notker der Reihe nach herauspräpariert werden." (Glauch 2000: 285).

10 Mit Abschnitten wird also auf Glauch (2000), gegebenenfalls in deren Zitierweise, z.B. auf Abschnitt 1 im 46. Kapitel des 1 . Buches als I 46.1., Bezug genommen und nicht auf die Abschnittszählung der lateinischen Ausgaben, vgl. Glauch (2000: 287).

11 Für die in den Text eingefügten lateinischen Wörter sind zur Unterscheidung von den eigentlichen Lehnwörtern im Deutschen verschiedene Termini gebraucht worden, von denen sich bislang keiner durchgesetzt hat. Es ist manchmal behelfsweise von andersprachigen „Einheiten“ und „Elementen“ (Riehl 2014: 24, 34, 208) die Rede. Kämmerer (2006) spricht von „Einschüben“ und meist von „Interkalationen“ (vgl. z. B. S. 245), Stolt (1964) von „Einschaltungen“ und „Umschaltungen“. Prinz (2010: 292) votiert für „Inserte“, was an Muyskens insertions erinnert, aber terminologisch nicht dem von diesem ebenfalls gebrauchten alternation gegenübergestellt ist, sondern diesen Fall eher einschließen soll. Ich verwende im Folgenden als vorläufigen Terminus „Einschub“, halte aber grundsätzlich auch Interkalation ebenso wie Insert für geeignet. Eine Zusammenfassung entsprechend dem Englischen foreign language item, etwa als ,fremdsprachiges Element', lässt sich jedoch schlecht handhaben. 


\subsection{Syntaktische Funktionen der lateinischen Einschübe}

Im Folgenden geht es nun darum, die Art, wie Notker Lateinisches in den Kontext des Althochdeutschen einbettet, an einigen Fallbeispielen genauer zu beschreiben. ${ }^{12}$ Dazu sollen die lateinischen Einschübe im Hinblick auf ihre grammatischen Formen und ihre Funktionen im Satzzusammenhang betrachtet werden. Das ist insbesondere vor dem Hintergrund der Diskussion um die Regeln der Einbettung fremdsprachlicher Elemente beim Code-Switching / Code-Mixing zentral.

In syntaktischer Hinsicht können die Einschübe mit Bezug auf ihren Satzgliedcharakter und ihre Funktion beurteilt werden. Die Einschübe können in verschiedenen Satzgliedfunktionen auftreten und dabei sowohl satzförmig als auch nominal sein. Sie können aber auch nur Satzgliedteile umfassen. Im Folgenden soll am Beispiel des bereits erwähnten 1. Kapitels gezeigt werden, wie sich die Einschübe diesbezüglich gestalten. ${ }^{13}$

\footnotetext{
(1) Fóne démo ér chît. Omnia uincit amor (3,13f.) ,von dem er sagt: Omnia vincit amor‘
}

Es handelt sich hier um einen Satz (Zitat) in direkter Rede in Objektfunktion, ganz ähnlich dem folgenden Beispiel (2):

(2) Fóne dír chád sî. Nate mee uires mea magna potentia solus $(4,13)$ ,Von dir sagte sie: Sohn, mein Kraft, meine große Macht bist du allein‘

Im folgenden Satz (3) nimmt eine lateinische NP (als Teil einer komplexen, koordinierten NP) die Position des Objekts ein, was ganz ähnlich für Satz (4) gilt, bei dem aber das verbindende dáz chît zwei lateinische NPs zusammenfügt.

(3) Tie ríngenten sâmen . dáz chît quatuor elementa . duíngest tû mít tóugenên bánden. $(3,16 / 17)$

,Die streitenden Urstoffe - das heißt die vier Elemente - fesselst du mit geheimnisvollen Banden'

(4) Ér méinet áber hîer epithalamia . dáz chît nuptialia carmina. (4,17/18)

,Er meint hier aber epithalamia, das heißt Hochzeitslieder.‘

12 Beispielzusammenstellungen aus Notkers Werken finden sich auch bereits in früheren Untersuchungen, z.B. Schaumann (1911), allerdings jeweils nach unterschiedlichen Kategorien und meist nach inhaltlich-sachlichen und nicht formalen Kriterien geordnet.

13 Die Textzitate werden nach King (1979) zitiert, d.h. nach Seite und Zeile in der Handschrift. Diese Angaben ermöglichen es auch, bei Glauch (2000), die die Manuskriptseiten angibt, die entsprechenden Übersetzungen zu finden, an die ich mich weitgehend gehalten habe. 
Im nächsten Beispiel sind sowohl die Subjekts- als auch die (komplexe) ObjektNP lateinisch.

(5) Tû bíst ter dén uirgilius héizet amorem . filium ueneris. (3,13)

,Du bist es, den Vergil Amor nennt, den Sohn der Venus.‘

Im folgenden Satz (6) findet sich das lateinische Element in der Subjekts-NP, die aber neben dem lateinischen Kern althochdeutsche Genitivattribute enthält. In diesem Satz lässt sich mit dem Beispiel natura auch die Abgrenzungsproblematik zum Lehnwort zeigen, da das öfter gebrauchte Substantiv natura an anderen Stellen aufgrund althochdeutscher Flexionsmorpheme eindeutig als Entlehnung eingestuft wird (vgl. AWB 2014: 6, 1075). ${ }^{14}$

(6) Mít lîebsamero gezúmfte . mít téro des cómenes únde der brûte natura gesíppôt uuírt $(4,4)$ ,durch ein angenehmes Bündnis, durch das die Naturen ${ }^{15}$ von Bräutigam und Braut ehelich verbunden werden

Satz (7) weist mehrere Typen lateinischer Einschübe auf. Zum einen besteht das Prädikativum aus einem lateinischen Substantiv, daneben weist die Subjekts-NP ein lateinisches Genitivattribut auf, und außerdem enthält der Gesamtsatz einen kompletten lateinischen Nebensatz in der Funktion einer Kausalangabe, die interessanterweise dem lateinisches Prädikatsnomen direkt nachgestellt ist.

(7) Apollinis lóbe-sáng héizent choreę. quia ipse pręest choris $(4,16)$

,Apollos Hymnen heißen choreae, weil er selbst die Chöre leitet.‘

Während insbesondere Objekt-NPs unter den lateinischen Einschüben häufig vertreten sind, stellen lateinische Präpositionalphrasen, wie im folgenden Satz (8)

14 Der vorliegende Beleg ist in AWB 2014: 6, 1078 als starkes Femininum unter der Bedeutung ,natürliche Beschaffenheit, von Menschen` eingeordnet. Das Fehlen des Akzents deutet darauf hin, dass für Notker (und den Schreiber) die Zuordnung zum Lateinischen dominierte. Glauch (2000) spricht von einem Fremdwort, zu dem in der vorliegenden Bedeutung kein ,ahd. Pendant“ (301) existiert und das „im Übergang zum Lehnwort“ (302) sei, vgl. Glauch (2000: 160). In den untersuchten Kapiteln tritt noch ein weiterer Beleg auf, der im Akk. Sg. tía natura (I 13,4/5) sicher keine lateinische Flexion aufweist, sondern wohl als morphologisch integriert anzusehen ist (vgl. AWB 2014: 6, 1075). King (1979: 13) konjiziert im Apparat „„natûra bzw. *naturam“.

15 Glauch (2000: 296) übersetzt hier mit einem Plural, wobei ich davon ausgehe, dass sie das für den deutschen Satz passender fand, aber nicht dass es im Lateinischen vorgegeben ist. 
als Lokalergänzung innerhalb eines attributiven verkürzten Nebensatzes (zu muoter) einen etwas selteneren Fall dar.

(8) tû dînero muoter zéizesto bíst. in papho ciuitate cypri sízzentero (4,9/10) ,der du der liebste deiner Mutter bist, die in Paphos, einer zyprischen Stadt, ihren Wohnsitz hat."

Schließlich sind noch die Einschübe im Eingangssatz zum ersten Kapitel zu besprechen. Dieser startet mit einer Anrede, einem lateinischen Namen im Vokativ, den das sprechende Subjekt, ebenfalls mit einem lateinischen Eigennamen benannt, anspricht.

(9) Himenee chît tiu satira $(3,8)$ „,Hymenäus, “spricht Satyra“

Hier zeigt sich einerseits, was schon die vorherigen Beispiele demonstriert haben, dass unter den lateinischen Einschüben häufig lateinische Eigennamen figurieren. Die Subjekts-NP besteht aber nicht nur aus einem lateinischen Eigennamen, der bereits im Prolog eingeführten Satyra, dieser wird von einem althochdeutschen bestimmten Artikel begleitet, was auch auf einen eher appellativischen Gebrauch deuten könnte. ${ }^{16}$

Dieser erste Überblick über die lateinischen Einschübe unter syntaktischem Gesichtspunkt zeigt, dass diese sehr vielfältig eingesetzt werden. Sie können sowohl ein ganzes Satzglied umfassen als auch nur einen Teil ausmachen, und zwar als Kern oder als Attribut. Die Einschübe können in nominaler Form, aber auch als Sätze erfolgen. Im ausgewählten Abschnitt erscheinen jedoch keine Beispiele für pronominale Einschübe. Auch ist in allen vorliegenden Fällen das Prädikat althochdeutsch. Bei einer Auswertung weiterer Passagen lassen sich aber auch komplexe Prädikate finden, wie in (10) und (11), bei denen die nicht konjugierte Form lateinisch ist.

(10) úbe síe in stige palude neuuúrtîn purificati $(73,2 / 3)$

,wenn sie nicht im Sumpf Styx gereinigt würden`

16 Glauch (2000: 296) ignoriert hier und auch an anderer Stelle (I 5,9) den Artikel bei der Übersetzung, was wohl darauf deutet, dass sie von einem Eigennamen ausgeht (die standarddeutsch keinen Artikel annehmen). An einer späteren Stelle (I 6,18), an der nochmals der Artikel im althochdeutschen Text auftaucht, übernimmt ihn Glauch auch in die Übersetzung. Der in der Tat auffällige Artikel kann vielleicht zumindest bei der ersten Nennung dadurch motiviert sein, dass er den anaphorischen Bezug auf den Prolog herstellen soll, in dem quędam satira eingeführt wird. Allerdings trägt der Artikel keinen auf demonstrativen Gebrauch deutenden Akzent. 
(11) Mág ter hímel transire . sô mag er óuh stare. $(33,6 / 7)$

,Kann der Himmel vergehen, kann er auch stehenbleiben.

Ein Sonderfall begegnet noch in Kapitel 2 (I 5,10), wo ein althochdeutscher Kausalsatz (vgl. Bsp. 15), einem Hauptsatz nach ne-uuéiz eingefügt ist, indem er mit der Abkürzung .s. für das lateinische Adverb scilicet ,nämlich` eingeleitet wird, womit eventuell der Charakter als Äußerungsbegründung unterstrichen wird. In der Regel verbindet s. ebenso wie .i. für id est , das heißt' lateinische Konstituenten, es lässt sich in den ersten Kapiteln aber auch noch ein weiteres Beispiel finden, wo .s. eine althochdeutsche Konstituente anschließt, in diesem Fall eine kausale althochdeutsche Präpositionalphrase (I 8,20). Aufgrund des sprachlichen Sondercharakters der Abkürzung werden diese Fälle in die folgenden Betrachtungen zunächst nicht mit einbezogen.

Unter den übrigen 112 lateinischen Einschüben bei Notker III., die im nächsten Kapitel genauer analysiert werden, sind die gemischten Konstituenten zumindest, wenn es sich um Einzelworteinschübe handelt, in der Mehrheit. Bei den Mehrworteinschüben machen die satzgliedwertigen lateinischen Phrasen drei Viertel der 23 Fälle aus, wobei hierunter auch die beiden oben erwähnten Objektsätze (1) und (2) sowie zwei Fälle von Nebensätzen gerechnet sind, die sich an ein den Hauptsatz abschließendes lateinisches Wort anfügen, wie in Beispiel (7) zu sehen. Dazu kommen acht rein lateinische Nominalphrasen (vgl. etwa Bsp. 5), und fünf Präpositionalphrasen, wie unten (26) und (27). Ein Beispiel für die Einbettung in gemischte Attributstrukturen ist unter (8) zu sehen sowie eine gemischte Mehrwort-Präpositionalphrase unter (28).

Wie gerade an den gemischten Konstituenten zu erkennen ist, lassen sich die lateinischen Einschübe in morphologischer Hinsicht weiter charakterisieren, mit Blick auf die grammatischen Kategorien, die im Kontext der althochdeutschen syntaktischen Umgebung gewählt werden und an denen die konkrete Art und Weise der Einbettung zu sehen ist. In Beispiel (10) weist das Partizip nach lateinischem Gebrauch eine Flexionsendung auf, die sich über ahd. sie als Mask. Pl. auf weiter vorausgehendes dii terrestres bezieht. Die ausgedrückten morphologischen Kategorien sind aber natürlich auch bei den rein lateinischen Konstituenten für die Beurteilung des Notker'schen Umgangs mit lateinischer und althochdeutscher Syntax von Interesse. 


\subsection{Morphologische Einzelanalysen}

\subsubsection{Einzelworteinschübe}

Im Folgenden soll anhand eines begrenzten Korpus, der ersten acht Kapitel der Martianus-Capella-Bearbeitung, in Einzelanalysen gezeigt werden, wie sich die lateinischen Einschübe in morphologischer Hinsicht charakterisieren lassen. ${ }^{17}$ Die Analyse wird getrennt für Einworteinschübe und für Mehrworteinschübe durchgeführt, da sich hier jeweils unterschiedliche Fragen stellen. Insgesamt sind die Einzelworteinschübe in diesem Teilkorpus mit 89 Vorkommen deutlich häufiger als die Mehrworteinheiten, die nur 23 betragen. Von den 89 Einzelwörtern gehört eine große Zahl, wie sich das auch schon an den obigen Beispielen sehen lässt, zu den Eigennamen, nämlich etwa 50. Bezüglich dieser Kategorie kann man sich fragen, ob man sie in gleicher Weise hier als lateinische Einschübe behandeln soll wie die Appellativa, da sie ja in der Regel gar nicht übersetzt werden können. Man kann daher die Eigennamen sicher nicht als Beleg für die Häufigkeit lateinischer Einschübe generell, sondern höchstens zur Charakterisierung einer bestimmten Textsorte, in der eben Eigennamen vorkommen, heranziehen. Andererseits sind die Eigennamen, v.a. die häufigen Personennamen, dennoch für die Frage, wie lateinische Wörter in den Kontext integriert werden, absolut einschlägig, da sie in gleicher Weise flexionsmorphologisch markiert sind und entsprechend ihrer syntaktischen Funktion dekliniert werden müssen. ${ }^{18}$ Abgesehen davon wäre die strikte Abgrenzung gegenüber Appellativa schwierig, da die Namen, wie etwa der der Göttin Memoria, oft auch eine appellative Bedeutung besitzen. Auffällig ist unter diesen Fällen die bereits erwähnte Verwendung eines weiblichen Personennamens mit definitem Artikel (tiu satira). Im Übrigen repräsentieren die in den ersten acht Kapiteln verwendeten Eigennamen überwiegend allein ein Satzglied, in einigen Fällen werden sie als Genitivattribute verwendet, wie etwa in Apollinis lóbe sáng in Satz (7). ${ }^{19}$ Daneben erscheinen sie öfter in gemischten Präpositionalphrasen, wie nâh euagrio in Satz (20), ze demo iúngen appolline in (24) und an weiteren Stellen, wie an argionam (I 9,2), nâh osiride

17 Bisagni (2013/2014: 22-23) berichtet über eine Pilotstudie, deren Ergebnisse erstaunlich gut zu denen seiner späteren, umfangreicheren Studie stimmen (40, Anm. 108). Das ist natürlich nicht generell gewährleistet, was die Aussagekraft der folgenden Ausführungen einschränkt.

18 Bisagni (2013/2014) bezieht die Eigennamen ebenfalls in seine Untersuchung zum altirischlateinischen Code-Switching ein, wobei deren Anteil in der von ihm untersuchten Glossierung deutlich niedriger ist, nämlich $7,7 \%$ beträgt.

19 Z.T. tritt der Genitiv auch in kongruierenden appositiven Fügungen auf wie etwa íro suéster mineruę $(11,5)$. 
(I 9,5) oder ze mantice (I 11,10). Soweit erkennbar, verhalten sich die Personennamen ganz den Appellativa analog, sodass ich mich im Folgenden auf die Appellativa konzentriere.

Unter den sicher appellativischen lateinischen Einzelworteinschüben (37) finden sich lediglich zwei Adjektive, im Übrigen handelt es sich um Substantive, wobei auch hier die Abgrenzung, etwa bei substantivierten Adjektiven, schnell problematisch wird. Die beiden Adjektive in den Beispielen (12) und (13) kongruieren mit vorausgehenden weiblichen Namen und weisen die syntaktisch geforderten lateinischen Flexionsendungen (Nom. bzw. Gen.) auf.

(12) íh méino dés . táz sî cęlestis íst (13,12) ,nämlich in Bezug auf ihre himmlische Herkunft

(13) dáz chît tero immortalis $(11,5)$

,d.h. der Unsterblichen`

Unter den Substantiven bilden satzgliedwertige Einzelworteinschübe in verschiedenen Kasus zwar die größte Gruppe (15 Fälle), mehrheitlich sind die Substantive allerdings in eine althochdeutsche Präpositionalphrase eingebunden (13-mal) oder sonst althochdeutsch erweitert (7-mal), entweder durch einen Artikel oder ein Genitivattribut. Überwiegend bilden die Einschübe also gemischtsprachige Konstituenten. In all diesen Fällen haben wir es mit Blick auf die Gesamtsätze mit einer althochdeutschen Matrixsprache zu tun, die die grammatische Struktur vorgibt, in die die lateinischen Elemente eingepasst werden. Bei den satzgliedwertigen Einzelworteinschüben scheint es aber, dass von der althochdeutschen Syntax her in einigen Fällen ein Artikel gefordert wäre, wobei hier eventuell auch personennamenartige Verwendungen eine Rolle spielen können. Oubouzar (1994: 83) geht jedenfalls von der Einbeziehung der lateinischen Substantive in das bei Notker schon relativ gefestigte Artikelsystem (Oubouzar 1994: 83) aus. Die folgenden Beispiele sollen die Verhältnisse illustrieren. Die Sätze (14) bis (17) zeigen verschiedene Satzgliedfunktionen der Einzelworteinschübe und damit verbunden verschiedene Kasus - mehrfach allerdings den Nominativ - die stets dem althochdeutschen Satzbauplan folgen, was grundsätzlich mit der Idee der Matrixsprache Althochdeutsch kompatibel ist.

(14) Sô gezímit animę . daz si intima sî sapientię... (12,19)

,So ziemt es sich für die Seele, wenn sie die Busenfreundin der Weisheit ist ${ }^{*}$

(15) uuánda ih peripatheticus pín (5,10/11)

.weil ich Peripatetiker bin“

(16) facundia nemág sîn mít libidine (15,11/12) ,Beredsamkeit kann nicht mit Begierde [...] einhergehen“

(17) Uuánda daz anima in mûot kenímet . táz kebíndet [...] memoria $(14,20)$ ,denn was die Seele wahrnimmt, das bindet die Erinnerung، 
Unter den übrigen Beispielen finden sich einerseits ähnliche Fälle, in denen die Einworteinschübe an der Subjektstelle stehen und lediglich zusätzlich mit einem althochdeutschen Artikel versehen sind, etwa im Plural, wie in Beispiel (18), oder ein komplexes Genitivattribut hinzutritt, wie in des cómenes únde der brûte natura in Beispiel (6).

(18) álso die palestrite gîengen $(10,12)^{20}$

,so wie die Ringkämpfer sich kleideten“

Während der Kasus durch die althochdeutsche Matrixstruktur vorgegeben wird, muss zumindest im Singular bei der Artikelsetzung auch jeweils das Genus des lateinischen inserierten Substantivs beachtet werden, wie etwa in Beispiel (19), wo der syntaktisch geforderte Genitiv im Sg. Mask. entsprechend dem Genus des lateinischen Substantivs zodiacus auftritt:

(19) dô si ín chátta . án der iâr-úmbe-uérte des zodiaci $(9,20)$

,als sie ihn beim Jahresumlauf des Zodiacus [...] begrüßte‘

Es gibt aber andererseits auch einige Fälle, in denen Inkongruenzen zu beobachten sind, wie etwa in Satz (20), in dem das maskuline Substantiv citharista zwar den maskulinen Artikel im syntaktisch geforderten Dativ aufweist, aber selbst nicht die entsprechende Dativform.

(20) nâh euagrio fáhende demo citharista $(7,14)$

,die dem Lyra-Spieler Euagrius [...] nacheiferten“

Hier ist wohl wie noch an weiteren Stellen eine Ablativform in einem den Dativ fordernden althochdeutschen Kontext statt einer Dativform gesetzt. ${ }^{21}$ Glauch (2000: 325) macht zu einer ähnlichen Textstelle mit dem weiblichen Personennamen berecinthia $(8,21 / 22)$ die Bemerkung, dass in einer ,deutsche[n] Absolutus-Konstruktion“ „der lat. Name im Ablativ“ stehe. Sie vergleicht diese Fälle mit der in der deutschen Sprachgeschichte bekannteren Erscheinung, dass bei Präpositionen, die den Dativ regieren, „lateinisch flektierte Wörter in den Ablativ“ (Behaghel 1924: 52) treten. Hierfür finden sich unter der Gruppe der Einzelwort-

20 Palestrite ist als Schreibung mit fehlender e caudata für die Pluralform palaestritae ,Ringkämpfer‘ aufzufassen, vgl. King (1979: 10, Komm. zur Stelle 12/13).

21 Stolt (1964: 277) spricht sich bei solchen hinsichtlich Nominativ oder Ablativ ambigen Formen ebenfalls für die Interpretation als Ablativ aus. Vgl. auch Beispiel (25). 
Einschübe in althochdeutschen Präpositionalphrasen (21-24) verschiedene Beispiele, etwa (23) sowie das unter (16) bereits zitierte mit libidine, während in (21) und (22) bezüglich Dativ und Ablativ ambige Formen erkennbar sind. Wie Satz (24) zeigt, fügen sich die Personennamen ganz in dieses Bild ein.

(21) under dien anderen pliadibus . dero sî éiniu íst. $(9,21)$

,unter den anderen Plejaden, deren sie eine ist ${ }^{\star}$

(22) Sîd er în hérebergónten sámint ímo an demo zodiaco [...] fúreréison ne-lâze. (16,3)

,da er ihn, der zusammen mit ihm am Tierkreis Quartier nimmt, nicht [...] vorauslaufen lasse

(23) daz mîn grâuua hóubet . únde fóne áltere zîtigez ze dero curia $(5,16)$ ,dass mein graues und vom Alter zur Kurie gereiftes Haupt

(24) Sî uuás áber dô îu [...] ze demo iúngen appolline gehîet. (11,15-17)

,Sie war aber da bereits [...] mit dem jungen Apollo verheiratet‘

Otto Behaghel (1924: 52) führt diesen Gebrauch darauf zurück, dass ein lateinischer Dativ „keine Präp. neben sich verträgt.“22 Die Vermutung, hier erscheine im althochdeutschen Text eventuell die von der entsprechenden lateinischen Präposition geforderte Kasusform, ${ }^{23}$ weist er mit Verweis auf den ebenfalls bei $z u$ und zwischen erscheinenden Ablativ zurück. Glauch (2000: 326) lehnt entsprechend auch die Interpretation der Ablative in den absoluten Dativkonstruktionen als „vom Lateinischen her gedacht“ oder als „lateinische ,Reservate““ ab. ${ }^{24}$ Eventuell ist mit einer bei der Präposition in erfolgten althochdeutschen Uminterpretation zu rechnen, die den Ablativ als vorgegebene Entsprechung übernahm (vgl. Bsp. 27). Es ist aber jedenfalls festzuhalten, dass hier ein Mismatch zwischen althochdeutscher Matrixsprache und der Grammatik des lateinischen Einschubs $\mathrm{zu}$ registrieren ist, der noch genauer zu analysieren ist.

22 Behaghel (1921: 136) führt zahlreiche Beispiele aus Notkers Schriften auf, darunter die oben unter (24) genannte Textstelle sowie ebenso den unter (20) erwähnten Fall, den er also auch durch die Präposition verursacht sieht, die den Eigennamen regiert, zu dem die betroffene Apposition gehört. Dafür spricht dasselbe Verfahren an einer vergleichbaren Stelle: fóne sînemo brûoder tiphone (9/8), wo ebenfalls der appositiv gebrauchte Eigenname im Ablativ steht. Dabei hebt Behaghel (1921: 136) hervor, dass er nur ein einziges Gegenbeispiel zu dem Verfahren Ablativ pro Dativ nach Präposition anführen könne (zi moysi Isid. 26,16).

23 Diese sich offenbar aufdrängende Annahme wurde auch von Teilnehmern an der Diskussion zu den Vorträgen vorgebracht. Vgl. dazu auch Anm. 29.

24 Zu Beispiel (23) nennt Glauch (2000: 308) die „Kommentarwendung morum maturitate curiae honore dignum“ als vermutliche Grundlage. Zumindest hier läge dann jedenfalls keine Vorprägung der Ablativform in (23) vor. 


\subsubsection{Mehrworteinschübe}

Die 23 lateinischen Mehrworteinschübe, in denen achtmal ein Eigenname enthalten ist, verteilen sich auf mehrere Typen. Zunächst fallen darunter auch die bereits genannten satzförmigen lateinischen Passagen, wie die Beispiele (1), (2) und (7). Mit Beispiel (8) ist ein weiterer Typ bereits angeführt worden: eine komplett lateinische Präpositionalphrase, die intern mehr oder weniger komplex sein kann. In (8) handelt es sich dabei um einen appositiv erweiterten Ortsnamen, der selbst in einen partizipial verkürzten Attributsatz eingebunden ist. Auch in Beispiel (25) ist die lateinische Präpositionalphrase in ein Attribut eingebunden, in dem sie der genaueren Lokalisierung der partizipial ausgedrückten Handlung dient.

(25) Táz îst éin bûoh apud grecos kescríbenez fóne dero apothesia $(6,7)$

,Das ist ein griechisches Buch über die Apotheose.

In den Sätzen (26) und (27) stehen die beiden lateinischen Präpositionalphrasen dagegen in Satzgliedfunktion.

(26) Uuánda íro uuíllo uuírt spûotigo gezúcchet . êina uuîla ad cęlestia . ánder uuîla ad terrestria. $(14,16 / 17)$

,denn ihr Wille wird schnell hin- und hergerissen, einmal zum Himmlischen, einmal zum Irdischen'

(27) Tóh si în [...] erslágenen fúnde in memphitica palude $(9,8)$

,Obwohl sie ihn [...] erschlagen im Sumpf von Memphis fand.‘

Neben lateinischen Präpositionalphrasen finden sich auch lateinische Mehrworteinschübe in Form verschieden attribuierter Nominalphrasen (durch Genitiv, Adjektiv oder Zahlwort), vgl. (29) sowie die oben bereits genannten Beispiele (3) bis (5). Die Nominalphrasen können ihrerseits auch in althochdeutsche Präpositionalphrasen eingefügt sein, wie in Beispiel (28):

(28) Uuánda fóne ęterna dei sapientia . íst animę sempiternitas kelâzen. (12,8/9)

Denn durch Gottes ewige Weisheit ist der Seele Unsterblichkeit gewährt.

(29) dáz íst tíu uuítta mít téro crines uirtutum zesámine-gechnúpfet uuérdent. (12,12/13) das ist das Band, mit dem die Haare der Tugenden zusammengebunden werden. 


\subsection{Zu Syntax und Morphologie der lateinischen Einschübe: ein erstes Fazit}

Auf der Basis der Sichtung der lateinischen Einschübe in den ersten acht Kapiteln der Notkerschen Martianus-Capella-Bearbeitung lassen sich folgende Feststellungen zusammenfassen.

1. Auch die angeführten Beispiele haben deutlich gemacht, dass es im Hinblick auf die beteiligten Wortarten keine große Variation gibt. Es handelt sich bei der Masse der untersuchten lateinischen Einschübe um nominale Elemente, seien es Einzelworteinschübe (ca. $77 \%$ ), bestehend aus Substantiven oder Eigennamen, oder Nominalphrasen in Gestalt von Mehrworteinschüben (ca. $11 \%)$. Die nächsthäufige Gruppe bilden die lateinischen Präpositionalphrasen mit gut $6 \%$. Es wurden keine in den althochdeutschen Text inserierten lateinischen Pronomina und Präpositionen oder Konjunktionen gefunden, auch keine Adverbien, und lediglich zwei Adjektive. Als Sonderfall lässt sich einzig die oben erwähnte lateinische Abkürzung .s. für das Adverb scilicet bei der Einleitung eines gemischtsprachlichen Nebensatzes bzw. einer althochdeutschen Präpositionalphrase anführen. Letztere Angaben beziehen sich natürlich nur auf Einzelworteinschübe, innerhalb von Mehrworteinschüben, wie Nebensätzen oder Präpositionalphrasen, finden sich auch Pronomina, Konjunktionen und Präpositionen.

2. Das Althochdeutsche kann für die kommentierenden Ausführungen Notkers durchweg als Matrixsprache gelten, die die grammatische Struktur vorgibt. Es gibt keinen Fall, in dem das flektierte Verb lateinisch ist. Es liegt also nicht der Fall eines schnellen Wechsels zwischen den Sprachen vor, der es verunmöglicht, für den Äußerungszusammenhang insgesamt anzugeben, was die Hauptsprache ist (Auer 1999: 315). In etwas mehr als der Hälfte der analysierten Sätze mit Code-Switching wird nur einmal innerhalb des Gesamtsatzes ins Lateinische gewechselt (35/61). Es sei denn, man bezieht in diese Überlegung mit ein, dass natürlich der Notker'sche Gesamttext auch noch rein lateinische Passagen enthält, die insgesamt dann doch einen stärkeren Eindruck eines sprachgemischten Textes ergeben. In den lateinischen Passagen sind im untersuchten Korpus aber keine althochdeutschen Einschübe zu finden, die Mischung erfolgt also nur in einer Richtung.

3. Es gibt nur wenige Fälle, in denen der Sprachwechsel an einer (Teil-)Satzgrenze stattfindet. Im Allgemeinen sind die lateinischen Einschübe in die althochdeutschen satzinternen Strukturen eingefügt, d.h., sie weisen in der Regel auch die Kasusmerkmale auf, die von der althochdeutschen Satzstruktur gefordert sind. Die Flexionsmorphologie selbst ist dabei soweit erkennbar immer lateinisch (abgesehen vom Sonderfall natura). Bei den satzgliedwerti- 
gen Einschüben sind davon keine Abweichungen zu beobachten. Allerdings kann es vorkommen, dass diese Einschübe lateinische Flexionsformen aufweisen, die zwar der althochdeutschen Syntax nicht widersprechen, aber nur von der lateinischen Syntax gefordert werden, etwa die Verwendung des Vokativs in Beispiel (9).

4. Wenn der Sprachwechsel innerhalb der Satzglieder stattfindet, was in den ersten acht Kapiteln ausschließlich Nominalphrasen und Präpositionalphrasen betrifft, so kann man behaupten, dass auch hier die Matrixsprache jeweils das Althochdeutsche ist, insofern der Wechsel bestimmten Mustern folgt. Gemischtsprachige Phrasen bestehen z.B. aus einer althochdeutschen Präposition oder einem althochdeutschen Definitartikel und einer lateinischen Nominalphrase, de facto meist einem Substantiv, vgl. etwa die Beispiele (16), (18), (19) sowie (28) mit einer lateinischen erweiterten NP. Das lateinische Substantiv kann auch mehrere althochdeutsche Bestandteile (Präposition, Artikel, Adjektiv) bei sich haben oder als Apposition zu einer althochdeutschen Phrase stehen (vgl. Anm. 18). Verbindungen von lateinischer Präposition und althochdeutscher Nominalphrase kommen jedoch ebensowenig vor wie lateinisches attributives Adjektiv vor althochdeutschem Substantiv. Bei Nominalphrasen mit Genitivattribut, etwa in den Beispielen (7) oder (19), stellt der lateinische Einschub überwiegend das Attribut dar. Lediglich in einem Fall (Bsp. 6) ist ein lateinisches Substantiv durch eine althochdeutsche Attributgruppe erweitert. ${ }^{25}$ Daran ist ersichtlich, dass grundsätzlich der Sprachwechsel zwischen Kopf und Genitivattribut in beide Richtungen erfolgen kann.

5. Dabei gibt es eine systematische Ausnahme von der Regel der matrixsprachlich vorgegebenen grammatischen Kategorien. Bei Präpositionalphrasen, die aus einer althochdeutschen Präposition mit Dativforderung und einer lateinischen Nominalphrase bestehen, stehen die Bestandteile der letzteren, d.h. die Substantive und auch allfällige Adjektive, wie in Beispiel (28) bei denjenigen lateinischen Klassen, in denen sich Dativ und Ablativ unterscheiden, im Ablativ. Ist das Substantiv von einem althochdeutschen Artikel sowie Adjektiv begleitet, wie in Beispiel (24), zeigt sich die Inkongruenz dann explizit zwischen der attributiven Erweiterung (im Dativ) und Substantivflexion (im Ablativ). ${ }^{26}$ Während sich hier also Kasusinkongruenz zeigt, erscheint ein

25 Dass das ausgerechnet bei dem hinsichtlich seines Status nicht ganz klaren Substantiv natura der Fall ist, könnte als Argument dafür genommen werden, dass es sich hier eben doch um ein Lehnwort handelt. Vor einer solchen Schlussfolgerung wären aber erst noch weitere Textpartien zu prüfen.

26 In gleicher Weise auch in einer Präpositionalphrase ohne Artikel: mít mísseliutigero pagina $(7,15)$. 
anderer möglicher Typ morphologischer Inkongruenz im untersuchten Material nicht: Wenn ein lateinisches Substantiv mit althochdeutschem Artikel und / oder althochdeutschem Adjektiv kombiniert ist, stimmt deren Genus durchweg überein.

Will man aus diesen Befunden Schlüsse für das Funktionieren der sprachgemischten Texte Notkers ziehen, muss man natürlich bedenken, dass die vorgelegte Analyse nur auf einem kleinen Ausschnitt der Notker'schen Textproduktion beruht. Die gewonnenen Erkenntnisse müssen durch weitere Beobachtungen, zunächst einmal in der vorhandenen Sekundärliteratur, ergänzt werden. So lässt sich der Ersatz Ablativ für Dativ in Präpositionalgruppen in der gesamten deutschen Sprachgeschichte beobachten und stellt eine Art Spezialgrammatik deutsch-lateinischer Texte dar. Lateinische Einschübe sind jedoch nicht generell auf Nominal- und Präpositionalphrasen beschränkt, gemischte Verbalphrasen lassen sich - wenn auch in geringer Zahl - bereits in den nicht ausgewerteten Passagen des Martianus Capella-Textes finden (vgl. Bsp. 10-11). Gemischte oder lateinische Verbalphrasen scheinen auch in anderen Notker-Texten, wie etwa der Boethius-Bearbeitung, beinahe ganz zu fehlen. ${ }^{27}$

Ebenso finden sich in Buch II der Martianus-Capella-Bearbeitung Fälle von Inkongruenzen bezüglich des Genus in althochdeutsch-lateinischen Nominalphrasen, etwa sélbêr ibis $(150,12)$, wo das lateinische feminine Genus nicht beachtet ist. ${ }^{28}$ Die Regel, dass eine lateinische Präposition nicht mit einem althochdeutschen Substantiv verbunden wird, bestätigt aber Näf (1979: 431) auch für die Notker'sche Bearbeitung von Boethius De consolatione philosophiae. Stolt (1964: 267) bestätigt das Fehlen der Kombination von althochdeutschem Substantiv mit lateinischem Adjektiv in Notkers Consolatio. Überhaupt weist Stolt (1964: 279) darauf hin, dass eine weitgehende Übereinstimmung zwischen Notkers und Luthers ,Mischprosa' bestehe, während Willirams Hoheliedparaphrase darüber hinausreichende Typen, wie etwa lateinische Verbalphrasen in althochdeutschem Kontext aufweise. ${ }^{29}$

27 Suzana Teodorović konnte in ihrer Hausarbeit im Rahmen des Seminars „Diglossie und Sprachmischung bei Notker von St. Gallen“ (FS 2013) zum ersten Buch von Notkers Consolatio nur drei Fälle gemischter Phrasen mit non-finiten Verbformen ermitteln.

28 Glauch (2000: 525) weist außerdem auf verschiedene Fälle von genusinkongruentem anaphorischen Bezug hin, etwa cęlestis ignis [...] dáz (62,21/22), mit neutraler Anapher zu einem lateinischen Femininum. Bekannt sind Fälle von Genusinkongruenz v. a. aus der Consolatio-Bearbeitung und den Psalmen, die Kelle (1890: 243-244) den Schreibern anlastet.

29 Das ähnelt den Verhältnissen bei den von Kämmerer (2006) untersuchten Predigten (2006: 224). 


\section{Notkers lateinisch-deutscher Sprachgebrauch in kontaktlinguistischer Perspektive}

In einem weiteren Schritt sollen nun die Beobachtungen, die an Notkers CapellaBearbeitung hinsichtlich der Verwendung lateinischer und althochdeutscher Bestandteile gemacht wurden, daraufhin untersucht werden, inwiefern sich die aus modernen Sprachkontaktsituationen bekannten Strategien und gewonnenen Charakterisierungen auch hier anwenden lassen. Unser Ausgangspunkt war ein Abrücken von der älteren Qualifikation von Notkers Sprache als ,Mischprosa‘, was teilweise, aber nicht grundsätzlich, mit einer negativen Konnotation verbunden war, und der Versuch, die Charakterisierung von Notkers Sprache als eine Ausprägung von Code-Switching zu prüfen. Das Abrücken von ,Mischprosa“ ist wohl auch wegen der Nähe zum Terminus Mischsprache angezeigt, der in der modernen Kontaktlinguistik auf eher seltene Fälle von Sprachen bezogen ist, die in spezifischen Konstellationen eine stabile ,gemischte' Struktur herausgebildet haben. ${ }^{30}$ Es ist aber ganz klar, dass Notkers Einsatz lateinischer Wörter jeweils einer Wahl geschuldet ist und diese Wörter in der Regel nicht obligatorische Bestandteile des Althochdeutschen sind, so dass Mischsprache keine adäquate Bezeichnung wäre. Die Inadäquatheit des Terminus Mischsprache für Notkers Sprachgebrauch ist auch eine Folge der Ambiguität des Begriffs ,Sprache‘, während mixed code, bilingual speech u.ä. klar darauf hindeuten, dass es nicht um die Charakterisierung einer Sprachstruktur, sondern um Rede, Sprechen (oder Schreiben) o.ä. geht, so dass ,gemischte Rede‘ oder ,gemischtes Schreiben“ adäquater wäre.

Der Umgang Notkers mit dem Lateinischen und Althochdeutschen scheint ansonsten zu dem stimmen, was die verschiedenen Autoren grundsätzlich als Code-Switching bzw. Code-Mixing analysieren. Das geschilderte Verfahren Notkers lässt sich etwa nach Myers-Scotton als Beispiel für die Verbindung einer Matrix language, die die grammatische Struktur und im Idealfall auch die grammatischen Morpheme vorgibt, und Embedded language-Elementen, die in diese grammatische Struktur eingebunden sind, vornehmlich lexikalische Einheiten, beschreiben. Insbesondere Mehrworteinheiten, die grammatische Morpheme der Embedded language und eine entsprechende interne Strukturierung aufweisen, werden dann als sogenannte Embedded language islands erfasst, wobei verschiedene Hypothesen im Hinblick darauf aufgestellt wurden, welche Morpheme aus

30 Dabei werden verschiedene Kriterien zur Definition diskutiert, vgl. zur Abgrenzung gegenüber Code-Mixing im Sinne Auers (1999: 321-328), der darüber hinaus auch noch fused lects unterscheidet. 
der Embedded language stammen können, etwa Pluralmorpheme, und welche aus der Matrix language stammen müssen, etwa Kasusmorpheme (z.B. MyersScotton \& Jake 2009). Gestützt auf Beispiele aus Luthers Tischreden (nach Stolt 1964) haben Auer \& Muhamedova (2005) das oben besprochene Verfahren Ablativ für Dativ bereits als Sonderfall einer Embedded language-Insel identifiziert, insofern hier der lateinische Kasus nicht mit der Kasusrektion der matrixsprachlichen Präposition korrespondiert. Auer \& Muhamedova (2005: 41) ziehen daraus den Schluss, hier liege eine durch die Embedded language beeinflusste Matrixsprache vor, womit sie gegen die strikte Trennung von Matrix language und Embedded language argumentieren, was hier nicht weiter verfolgt werden soll. ${ }^{31}$ Eine zusammenfassende Beurteilung der Rolle solcher Inkongruenzen in Notkers Sprachproduktion, zu denen dann zumindest auch noch Fälle von Genusinkongruenz, wie oben erwähnt, gehören, wäre vor der Analyse umfangreicherer Textpassagen und weiterer Texte verfrüht. Die weitgehende Übereinstimmung mit dem Modell einer Matrix language mit Embedded language-Elementen, insbesondere Embedded-islands, die ihre eigene interne Morphologie bewahren, bedeutet auch, dass Notkers Sprachgebrauch in Muyskens Terminologie am ehesten als insertion $\mathrm{zu}$ beschreiben ist, wobei unter den Mehrworteinschüben gelegentlich auch alternation vorkommt. Bezieht man in die Gesamtbetrachtung mit ein, dass Notkers Text neben den hier untersuchten intrasententiellen Sprachmischungen zahlreiche Wechsel von lateinischen und althochdeutschen Sätzen enthält, erweist sich der Alternations-Typ durchaus auch als adäquate Beschreibung des Notker'schen Schreibgebrauchs.

Allerdings ist noch $\mathrm{zu}$ diskutieren, inwiefern hier Beispiele einer anderen kontaktlinguistischen Erscheinung vorliegen, die jüngst Maarten Kossmann (2010) als Parallel system borrowing analysiert hat. Kossmann illustriert die Erscheinung zunächst mit den bekannten bildungssprachlichen Pluralformen, etwa engl. alumnus vs. alumni, weist aber nach, dass Parallelparadigmen durchaus weiter verbreitet sind. Als ein weiteres Beispiel eines solchen Parallel system borrowing führt er lateinische Flexionsformen bei biblischen Namen aus dem Frühneuhochdeutschen (Hans Sachs) an (Kossmann 2010: 465-466), die den oben

31 Zweifellos kann neben Code-Switching auch strukturelle Beeinflussung in einem sprachgemischten Satz vorliegen; fraglich ist, ob die beiden Erscheinungen voneinander unabhängig sind, wogegen sich Auer \& Muhamedova (2005: 47) wenden. Im konkreten Fall beruht ihre Interpretation der frühneuhochdeutschen Beispiele auf der Annahme, die lateinische Kasusrektion richte sich nach der entsprechenden lateinischen Präposition, was nach Behaghel (1924), wie oben ausgeführt, vgl. Anm. 21, eher unwahrscheinlich ist. Generell gilt aber Muyskens Feststellung „In many bilingual communities code-mixing goes hand in hand with structural convergence.“ (2000: 123) 
diskutierten Beispielen Notkers strukturell absolut entsprechen, etwa Pilati weib in Beispiel (7). Die traditionelle Lehnwortforschung, die sich mit der Analyse der materiellen Entlehnungen (mat-loans) beschäftigt, hat diesem ,paradigmatic borrowing restricted to loanwords“ (2010: 461) zweifellos wenig Bedeutung beigemessen, was Kossmann (2010: 460) auf „the similarity (at least in its results) with code-switching“ zurückführt, das sich eben gerade auch durch den Erhalt der fremdsprachlichen Morphologie (Embedded language-Morphologie) auszeichne. Kossmann (Kossmann 2010: 482) kann an einer Reihe von Beispielen überzeugend darlegen, dass es Parallelparadigmen gibt, die in den Bereich der Entlehnung gehören, da die Quellsprachen beispielsweise gar nicht mehr beherrscht werden und so Code-Switching nicht in Frage kommt. Sein Fokus liegt auf dem Nachweis, dass es hier um morphologische Entlehnung geht; die Abgrenzung gegenüber Code-Switching erfolgt nur indirekt, etwa durch Bemerkungen, dass es nicht um „occasional insertions“ (Kossmann 2010: 482) gehe, sondern offenbar um die Eigenschaft von Lexemen. Er weist aber darauf hin, dass Code-Switching eine Rolle bei der Herausbildung solcher Parallelparadigmen gespielt haben könne und spekuliert, ,that it is a form of petrified code-switching“ (Kossmann 2010: 482). Er geht jedenfalls davon aus, dass zur Entstehung des Parallel system borrowing ein gewisser Bilingualismus, jedenfalls Kenntnis dieser Paradigmen, vorausgesetzt werden müsse. Gerade für die Fälle von gelehrten Parallelparadigmen, wie bei den lateinischen Flexionsparadigmen im Englischen und im Deutschen, nimmt er an, dass sie ein Produkt der Schriftlichkeit seien, was seines Erachtens gegen Code-Switching spreche. Es scheint, dass Kossmann damit eventuell schriftliches Code-Switching ausschließen möchte oder jedenfalls Fälle wie die bei Notker behandelten, die den von ihm aus Hans Sachs zitierten Beispielen sehr ähnlich sind, dennoch eher nicht als Code-Switching qualifizieren würde. Er führt aber auch andere Fälle an, in denen die vorgefundenen entlehnten Paradigmen keine genaue Parallele in aktuellem Code-Switching hätten (Kossmann 2010: 482), sondern z. B. nur „quasi-Arabic noun formation“ darstellten. Generell hält er fest, dass „PSB does not necessarily imply that the borrowed elements are fully identical to the source" (Kossmann 2010: 461), was bei Code-Switching eher zu erwarten ist, da hier ja nach den meisten Definitionen in einen anderen korrekten Code gewechselt wird. Das Verhältnis von Code-Switching und der Entstehung paralleler morphologischer Inventare muss also vorerst noch offenbleiben.

Schließlich soll noch der Frage nach dem schriftlichen Code-Switching nachgegangen werden. $\mathrm{Ob}$ man das schriftliche Vorkommen von „,cases where lexical items and grammatical features from two languages appear in one sentence“, wie Muysken definiert (vgl. Abschnitt 2), ebenfalls zum Code-Switching zählen soll, ist vielleicht auch ein terminologisches Problem. Dass solche Sätze vorkommen, ist nicht nur in der obigen Analyse, sondern auch in anderen Untersuchungen 
bereits gezeigt worden. Was nicht geklärt ist, ist, ob diese Konstruktionen Abbild eines mündlichen Gebrauchs sind oder ob es auch ein spezifisch schriftliches Code-Switching gibt. In jedem Fall ist es aber möglich und auch nötig, die Verfahren, die beim Gebrauch von Elementen zweier Sprachen im mündlichen Gebrauch und in geschriebenen Texten vorkommen, zu beschreiben und auf ihre Vergleichbarkeit hin $\mathrm{zu}$ analysieren. Wir haben oben gesehen, dass eine Zuordnung der Notker'schen Verfahren zu dem, was wir über Code-Switching / Codemixing wissen, grundsätzlich naheliegt. Wo sich hier im Detail Abweichungen ergeben, kann aber erst auf der Basis einer umfassenderen Untersuchung bestimmt werden.

Einen Vergleich der Ergebnisse verschiedener Studien im Hinblick auf die von Code-Switching betroffenen Konstituenten hat Herbert Schendl (2000) unternommen. Seine eigenen Untersuchungen zum lateinisch-englischen Code-Switching in zwei Textsorten basieren auf je einem etwa doppelt so umfangreichen Korpus wie dem hier untersuchten Notker'schen Teilkorpus. Beim Vergleich der Kategorien von Einschüben (,switched constituents“) zeigen sich große Unterschiede in der Häufigkeit, nicht nur gegenüber den modernen Verhältnissen, sondern auch zwischen der untersuchten Dichtung und den Predigten, die durch lateinische Matrixsprache gekennzeichnet sind. Interessant sind insbesondere die Diskrepanzen bei den „single word switches“, die bei den Predigten ähnlich wie in den modernen Daten mehr als 20\% ausmachen, während sie in den Gedichten fast gar nicht vorkommen (<3\%). Dafür ist dort der Anteil vollständig lateinischer NP-Einschübe („switched noun phrases“, Schendl 2000: 80) höher als in allen herangezogenen Vergleichskorpora, nämlich 30\%, was Schendl (2000: 79) auf viele „prefabricated chunks“ zurückführt. Der Anteil der Präpositionalphrasen scheint generell in den historischen Texten etwas höher zu sein (ca. 14\%). ${ }^{32}$ Schendl bestätigt die schon andernorts gemachte Feststellung, dass „switching between major constituents (NP, VP, PP) over switching within these major constituents“ (2000: 77) dominiert. ${ }^{33}$ Die Ergebnisse der vorliegenden Analyse

32 Die Aufschlüsselung der internen Verhältnisse zeigt, dass Schendl hier nicht nur rein lateinische Präpositionalphrasen zusammenfasst, sondern auch gemischte Phrasen subsumiert, die allerdings auch bei den Predigten stark in der Minderheit sind, vgl. Schendl (2000: 78). Unklar bleibt dabei, ob sich hier auch Einzelworteinschübe verbergen, da bei deren Behandlung (2000: 79-80) keine Beispiele mit Substantiveinschüben innerhalb einer Präpositionalphrase genannt werden. Hier zeigt sich, dass in der Code-Switching-Forschung Zählungen verschiedener Autoren teilweise im Einzelnen schwer vergleichbar sind, was man auch an den verschiedenen Leerstellen in Schendls Tabelle sieht, wo er sich auf die modernen Untersuchungen bezieht.

33 Allerdings ist mir unklar, woraus sich die bei Schendl (2000: 77) genannte Zahl von 94\% bzw. $82 \%$ ergibt. 
sind diesbezüglich nicht ganz eindeutig. Immerhin sind bei den Einzelworteinschüben, auch unter Einbeziehung der Personennamen, leicht überwiegend gemischte Phrasen betroffen (18 gegenüber 16, s.o., sowie etwa die Hälfte bei den Eigennamen). Bei den Mehrworteinschüben, bei denen definitionsgemäß auch Sätze (im Sinne von clauses) betroffen sind, stehen sich im Übrigen jedoch 13 rein lateinische Konstituenten und nur sechs gemischte gegenüber. Dass es für die schriftlichen Texte charakteristisch ist, dass ihnen die „emblematic switches“ (Schendl 2000: 78) durch Gesprächspartikeln und Interjektionen fehlen, lässt sich auch mit den vorliegenden Ergebnissen zu Notker bestätigen.

Aufgrund der zeitlichen Nähe, der identischen Gebersprache und der vergleichbaren Funktionalität von Glossierung zum besseren Textverständnis und der Notkerschen Unternehmung der übersetzenden und kommentierenden Texterläuterung ist es von besonderem Interesse, die Ergebnisse von Jacopo Bisagnis Studie (2013/2014) zum Altirischen zu vergleichen. In der großen Mehrheit der Fälle handelt es sich um die Einbettung lateinischer Elemente in die Matrixsprache Altirisch, was Bisagni als Reflex eines „common linguistic behavior in early medieval Irish monasteries“ (2013/2014: 37) sieht, der den Texten automatisch die Konnotation eines Produkts einer intellektuellen Elite verleihe bzw. als „manifestation of an in-group mode of discourse“ (56) angesehen werden könne. Diese Qualifikationen, die vielleicht in ähnlicher Weise für das Kloster St. Gallen angenommen werden können, müssen bezüglich des Notker'schen Sprachgebrauchs hinsichtlich der schulischen Situation perspektiviert werden, wo in einem weiteren Sinn aber sicher auch von einem sprachlichen In-groupVerhalten ausgegangen werden kann. Im Detail zeigen sich einige weitere Charakteristika, die mit Notkers Sprachgebrauch tendenziell vergleichbar sind, insbesondere dass die lateinischen Einschübe mit lateinischer Flexionsmorphologie versehen sind, was sie zu Embedded language islands macht. Dabei machen die Substantive sowie Nominalphrasen ebenfalls die Mehrheit der intrasententiellen Einschübe aus (52,1\%), eine zweite große Gruppe stellen die Präpositionalphrasen (28,1\%), während Verben (Partizipien, Infinitive) und Pronomina nur zu 1,6\% und Adjektive zu 4,2\% als Einschübe vorkommen sowie Präpositionen gar nicht (2013/2014: 39). Auch wenn die Prozentzahlen im Einzelnen nicht dem untersuchten Notker'schen Teilkorpus übereinstimmen, ist es einzig die große Zahl an Adverbien (9,9\%), die im Notker'schen Korpus nur sporadisch (als Abkürzungen) erscheinen, was wirklich auffällig ist. Ansonsten sind die Kategorien der Einschübe tendenziell ähnlich verteilt. Außerdem verzeichnet Bisagni (2013/2014: 39) auch zwei lateinische Numeralia, während solche im hier untersuchten Notker-Korpus als Einzelworteinschübe völlig fehlen. Dabei könnte aber einer der beiden von Bisagni angeführten Fälle aus den Würzburger Glossen bei anderer Klassifikation eventuell als Mehrworteinschub gewertet 
werden. ${ }^{34}$ Bei solchen Vergleichen muss also unbedingt beachtet werden, dass Unterschiede in den Anteilen auch auf unterschiedliche Zählmethoden zurückzuführen sein können, weshalb es wichtig ist, dass die Analyse, wie bei Bisagni (2013/2014), nachvollziehbar gemacht wird. Abgesehen von den quantitativen Unterschieden bei den betroffenen Wortklassen und Konstituenten erscheint noch eine weitere interessante Abweichung gegenüber dem Notker-Korpus. Bisagni gibt an, dass in seinem Korpus nur zwei Nomina (1,9\%) mit Ablativformen vorkommen, während die Mehrheit der lateinischen Einschübe Nominativ Singular-Morphologie aufweist (2013/2014: 41), was er mit den Sonderfunktionen des Ablativs erklärt. Hier wäre eine genauere Betrachtung der betroffenen Kasusformen von Interesse.

\section{Schlussbemerkung}

Bisagni (2013/2014: 52) zieht aus seiner Untersuchung insgesamt den Schluss, dass der Vergleich des Sprachwechsels in den Glossen mit modernem CodeSwitching angebracht und lohnend sei, nicht nur weil man Aufschlüsse über vergleichbare Mechanismen in der älteren Schriftlichkeit erhalten kann, sondern auch, weil die Code-Switching-Forschung damit um spezifische Fälle von Sprachpaaren im Kontakt bereichert werden kann. Ähnlich hatte schon Schendl (2000: 70-71) auf die Notwendigkeit der Untersuchung historischer Texte hingewiesen, um diachrone Aspekte des Code-Switchings auch in die allgemeine linguistische Diskussion einbringen zu können. Im Hinblick auf den Sprachgebrauch Notkers lässt sich noch kein abschließendes Urteil fällen. Unsere Pilotstudie hat zumindest keine Fälle ans Licht gebracht, die gänzlich den aus modernem Code-Switching bekannten Verhältnissen widersprechen, wenn auch die bewahrten Kasusmorpheme der Embedded language bei den Einworteinschüben nicht ganz in Bild passen und jedenfalls den Behauptungen von Poplack \& Dion (2012) zu widersprechen scheinen. Grundsätzlich ist es durchaus denkbar, dass sich hinter Notkers schriftlichem Code-Switching mündlich im Kloster gebrauchte Strategien verbergen. Eine solche Annahme ist aber auch nicht zwingend, und es könnte sich folglich auch um spezifisch schriftliche Verfahren handeln, die dann vielleicht als typisch schriftliches Code-Switching klassifiziert werden könnten. Erst weitere und umfangreichere Analysen der Notker'schen Texte können Aufschluss

34 Es handelt sich um die Stelle Wb 11a10: unus accipit palmam. Das lateinische Zitat ist in einen altirischen Kausalsatz eingebettet und fungiert m.E. dort als Subjekt. Das mit unus kongruierende flektierte Verb wird jedenfalls von Bisagni nicht bei den Verb-switches verzeichnet. 
darüber geben, welche Verfahren für Notkers verschiedene Texte wirklich typisch sind. Es ist daher zu hoffen, dass sich weitere Untersuchungen Notkers reichem Sprachmaterial annehmen.

\section{Literatur}

AWB = Althochdeutsches Wörterbuch, begr. v. Elisabeth Karg-Gasterstädt und Theodor Frings, hrsg. von Hans Ulrich Schmid (2015), Bd. 6, Berlin / Boston, 2015.

Auer, Peter (1999), „From codeswitching via language mixing to fused lects. Toward a dynamic typology of bilingual speech“, in: International Journal of Bilingualism, 3, 309-332.

Auer, Peter / Raihan Muhamedova (2005), ,,Embedded language“ and ,matrix language“ in insertional language mixing“, in: Italian Journal of Linguistics. Rivista di Linguistica, 17, 35-54.

Baesecke, Georg (1950), Vor- und Frühgeschichte des deutschen Schrifttums, Bd. 2: Frühgeschichte, 1. Lieferung, Halle a.d.S.

Behaghel, Otto (1921), „Deutsche Präposition mit lateinischer Casusform“, in: Beiträge zur Geschichte der deutschen Sprache, 45, 136-137.

Behaghel, Otto (1924), Deutsche Syntax, Bd. 2: Die Wortklassen und Wortformen, B. Adverbium, C. Verbum, (Germanische Bibliothek, 1: Grammatiken, 10), Heidelberg.

Bisagni, Jacopo (2013-2014), „Prolegomena to the study of code-switching in Old Irish glosses“, in: Peritia, 24-25, 1-58.

Bulitta, Brigitte (2014), „Von abrizza über libs bis modul. Zum Lehnwortschatz im Althochdeutschen und seiner Behandlung im Althochdeutschen Wörterbuch (Thesaurus)“, in: Andrea Bambek \& Volker Harm (Hrsg.): Fremd- und Lehnwortschatz im sprachhistorischen Wörterbuch, (Germanistische Linguistik, 228), Hildesheim, 17-50.

Ekkehart = Casus St. Galli, Ekkehardi IV. Casus Sancti Galli. St. Galler Klostergeschichten, (Lateinisch-Deutsch), Hans F. Haefele (Hrsg.) (1991), Darmstadt.

Glauch, Sonja (2000), Die Martianus-Capella-Bearbeitung Notkers des Deutschen, Bd. 1: Untersuchgen, Bd. 2: Übersetzung von Buch 1 und Kommentar, (Münchener Texte und Untersuchungen zur deutschen Literatur des Mittelalters, 116-117), Tübingen.

Grotans, Anna (1998), „Simplifying Latin“, in: Journal of Germanic linguistics and literatures, 19, $1-43$.

Grotans, Anna (2006), Reading in Medieval St. Gall, (Cambridge Studies in Paleography and Codicology, 13), Cambridge.

Hoffmann, Paul (1910), Die Mischprosa Notkers des Deutschen, (Paleastra, 58), Berlin.

Kämmerer, Carmen Maria (2006), Codeswitching in Predigten des 15. Jahrhunderts. Mittellatein Frühneuhochdeutsch. Mittellatein - Altitalienisch, (Studies in eurolinguistics, 4), Berlin.

Kelle, Johann (1890), „Die St. Galler deutschen Schriften und Notker Labeo“, in: Abhandlungen der Königlich Bayerischen Akademie der Wissenschaften, Classe 1, Bd. 18, Abt. 1, München, 206-280.

Die Werke Notkers des Deutschen. Neue Ausgabe, begonnen von Edward H. Sehrt und Taylor Starck, fortgesetzt von Peter W. Tax und James C. King, Bd. 4: James C. King (Hrsg.) (1979), Notker der Deutsche: Martianus Capella ,De nuptiis Philologiae et Mercurii‘, Tübingen.

Kossmann, Maarten (2010), Parallel System Borrowing: Parallel morphological systems due to the borrowing of paradigms, in: Diachronica, 27, 459-487. 
Luginbühl, Emil (1970), Studien zu Notkers Übersetzungskunst. Mit einem Anhang: Die Altdeutsche Kirchensprache, Einleitung von Stefan Sonderegger, (Das Althochdeutsche von St. Gallen, 1), Berlin.

Muysken, Pieter (2000), Bilingual speech: a typology of code-mixing, Cambridge.

Myers-Scotton, Carol \& Janice Jake (2009), „A Universal Model of Codeswitching and bilingual language processing and production“, in: Barbara E. Bullock \& Almeida Jacqueline Toribio (Hrsg.), The Cambridge Handbook of Linguistic Code-switching, Cambridge, 336-357.

Myers-Scotton, Carol (2010), Contact linguistics: bilingual encounters and grammatical outcomes, Oxford.

Näf, Anton (1979), Die Wortstellung in Notkers Consolatio. Untersuchungen zur Syntax und Übersetzungstechnik, (Das Althochdeutsche von St. Gallen, 5), Berlin.

Poplack, Shana \& Nathalie Dion (2012), „Myths and facts about loanword development“, in: Language Variation and Change, 24, 279-315.

Poplack, Shana (2013), „The second decade (1973-1983)“, in: Linguistics, 51, 11-14.

Prinz, Michael (2010), ,Vergessene Wörter - frühe volkssprachige Lexik in lateinischen Traditionsurkunden“, in: Markus Hundt \& Alexander Lasch (Hrsg.), Deutsch im Norden. Varietäten des norddeutschen Raumes, (Jahrbuch für Germanistische Sprachgeschichte, 6), Berlin / New York, 292-322.

Riehl, Claudia Maria (2014), Sprachkontaktforschung. Eine Einführung. 3., überarb. Aufl. Tübingen.

Schaumann, Ernst (1911), „Studien zu Notkers Mischprosa“, in: Jahresbericht des Gymnasiums der k.k. Theresianischen Akademie in Wien, 3, 3-40.

Schendl, Herbert (2000), „Syntactic constraints on code-switching in medieval texts“, in: Irma Taavitsainen / Terttu Nevalainen / Päivi Pahta \& Matti Rissanen (Hrsg.), Placing Middle English in Context, Berlin / New York, 67-86.

Schendl, Herbert (2012), „Literacy, Multilingualism and Code-switching in Early English Written Texts“, in: Mark Sebba / Shahrzad Mahootian \& Carla Jonsson (Hrsg.), Language mixing and code-switching in writing. Approaches to mixed-language written discourse, (Routledge Critical Studies in Multilingualism), London, 27-43.

Sebba, Mark (2012), „Researching and Theorising Multilingual Texts“, in: Mark Sebba / Shahrzad Mahootian \& Carla Jonsson (Hrsg.), Language mixing and code-switching in writing: approaches to mixed-language written discourse, (Routledge Critical Studies in Multilingualism), London, 1-26.

Skaffari, Janne \& Aleksi Mäkilähde (2014), Code-Switching in Historical Materials: Research at the Limits of Contact Linguistics, in: Robert Nicolaï (Hrsg.), Questioning Language Contact. Limits of Contact, Contact at its Limits, (Brill Studies in Language Contact and Dynamics of Language, 1), Leiden / Boston, 252-279.

Sonderegger, Stefan (2003), Althochdeutsche Sprache und Literatur, 3. Aufl. Berlin / New York. Stolt, Birgit (1964), Die Sprachmischung in Luthers „Tischreden“. Studien zum Problem der Zweisprachigkeit, (Acta Universitatis Stockholmiensis, Stockholmer germanistischer Forschungen, 4), Stockholm.

Oubouzar, Erika (1992), „Zur Ausbildung des bestimmten Artikels im AHD“, in: Yvon Desportes (Hrsg.), Althochdeutsch. Syntax und Semantik. Akten des Lyonner Kolloquiums zur Syntax und Semantik des Althochdeutschen. (1.-3. März 1990), (Serié germanique ancien, 1), Lyon, 69-87. 